\title{
OBIC Analysis of Stressed, Thermally-Isolated Polysilicon Resistors
}

\author{
Edward I. Cole Jr., †John S. Suehle, Kenneth A. Peterson, *Prasad Chaparala, \\ Ann N. Campbell, Eric S. Snyder, and Donald G. Pierce \\ Electronics Quality/Reliability Center \\ Sandia National Laboratories, MS 1081 \\ Albuquerque, New Mexico 87185-1081 \\ +National Institute of Standards and Technology, Gaithersburg, MD 20899-0001 \\ *University of Maryland, College Park, MD 20742
}

\begin{abstract}
High gain Optical Beam Induced Current (OBIC) imaging has been used for the first time to examine the internal structural effects of electrical stress on thermally-isolated polysilicon resistors. The resistors are examined over a wide range of current densities, producing Joule heating up to $\sim 1200{ }^{\circ} \mathrm{C}$. Throughout this current density range, the OBIC images indicate a clustering of dopant under $\mathrm{dc}$ stress and a more uniform distribution under ac conditions. The OBIC images also reveal areas that are precursors to catastrophic resistor failure. In addition to OBIC imaging, conventional electrical measurements were performed, examining the polysilicon resistance degradation and time-to-failure as a function of electrical stress. The electrical measurements show a monotonic increase in polysilicon resistor lifetime with frequency (up to $2 \mathrm{kHz}$ ) when subjected to a bipolar ac stress. The enhanced lifetime was observed even under high temperature (from Joule heating) stress conditions previously reported to be electromigration-free [1]. The dopant redistribution indicated by the OBIC images is consistent with an electromigration stress experienced by the polysilicon resistors. The implications for thermallyisolated polysilicon resistor reliability are examined briefly.
\end{abstract}

\section{INTRODUCTION}

Polysilicon interconnections are used throughout the microelectronics industry. In addition to integrated circuit (IC) uses, polysilicon resistors have found applications as micro-heaters, spectrometer infrared light sources, and temperature sensing elements $[1,2]$. The stability and reliability of polysilicon are issues at high frequencies, high current densities, and elevated operating temperatures. Electrical stress on polysilicon resistors is normally considered to be a function of current density and temperature. At high current densities $\left(\geq 10^{6} \mathrm{~A} / \mathrm{cm}^{2}\right)$ electromigration effects have been reported with accompanying resistance changes attributed to redistribution of dopant species in encapsulated resistors [3]. Resistance variations have also been observed at lower current densities $\left(-10^{5} \mathrm{~A} / \mathrm{cm}^{2}\right)$ in thermally-isolated, micromachined resistors [2, 4]. A large initial drop in resistance is also observed during stressing of thermally- isolated polysilicon resistors at high Joule heating temperatures. Two proposed mechanisms for the resistance decrease are recrystallization and subsequent polysilicon grain growth [1] and grain boundary dopant segregation [5] during self-heating. Earlier reports found no evidence of electromigration for thermally-isolated, stressed resistors .[1]. For each of these mechanisms (electromigration and high temperature stress), a local variation of the polysilicon Fermi level is produced.

We have, for the first time, applied high gain OBIC [6, 7] imaging to thermally-isolated polysilicon resistors to analyze the distribution of Fermi level changes for different stress conditions. The physics of OBIC signal generation in polysilicon are described. The OBIC data acquisition system is also discussed. The OBIC results illustrate how the internal structural changes in polysilicon resistors can be directly observed over a wide range of stressing conditions. Electromigration effects at high temperature $\left(\sim 1100^{\circ} \mathrm{C}\right)$ are clearly seen in the OBIC images. Resistance degradation and lifetime measurements are also reported and are shown to agree well with the OBIC imaging results.

\section{POLYSILICON RESISTOR STRUCTURE}

The polysilicon resistors were fabricated in a MOSIS foundry service using a $2.0 \mu \mathrm{m}$ double metal, double polysilicon, $n$-well CMOS process [8]. A post-process etch step was used to remove the exposed silicon substrate material to create the suspended resistor structure [4]. Fig. 1 shows a schematic cross section of a resistor. The resistor structure is a sandwich of field and CVD oxides that encapsulate a polysilicon resistor. The $4000 \AA$ thick, $4 \mu \mathrm{m}$ wide, CVD polysilicon film is doped in-situ with phosphorous, resulting in a sheet resistance of $21 \Omega / s q$. A scanning electron micrograph of a thermally-isolated polysilicon resistor is shown in Fig. 2.

\section{CONVENTIONAL StREsS TESTING REsults}

Life tests were conducted by applying dc, bipolar, or unipolar currents with an HP 3245A universal source or an HP8116 function generator. Failure was defined as a tenfold 
increase from the initial resistance. The devices were stressed either in ceramic packages or as die on a probe station. The stress was removed during resistance measurement.

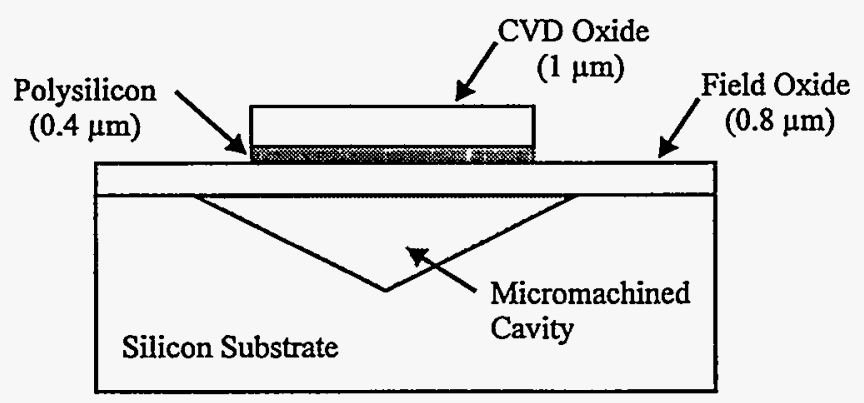

Fig. 1. Schematic cross section of a thermally-isolated polysilicon resistor.

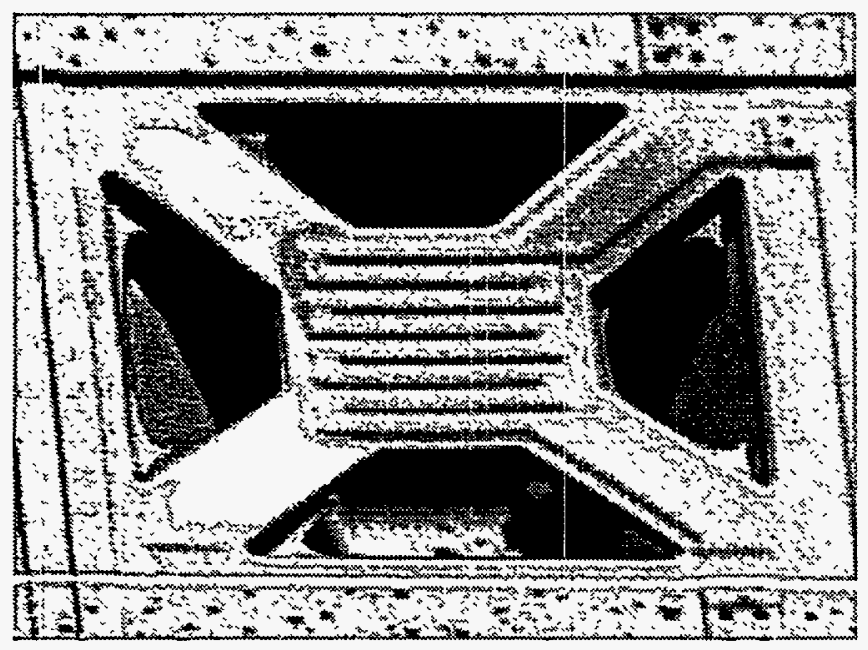

Fig. 2. SEM micrograph of a polysilicon resistor.

\section{Stressing}

Fig. 3 displays the variation in resistance resulting from constant current dc stressing. To determine the resistance, the voltage was measured with $0.1 \mathrm{~mA}$ applied current. Two features stand out in Fig. 3. First, there is an initial drop in resistance that occurs for high stress current density. The initial drop in resistance is proportional to the stress current density, but does appear to saturate at high current densities $\left(2.2 \times 10^{5}\right.$ and $2.1 \times 10^{5} \mathrm{~A} / \mathrm{cm}^{2}$ have similar resistance drops). One mechanism proposed for the reduction in resistance is polysilicon recrystallization and subsequent grain growth when the Joule heating is in excess of $800^{\circ} \mathrm{C}$ [9]. For a given dopant concentration, it has been shown that the polysilicon resistivity decreases with increasing grain size [10]. A second possible mechanism for the reduced resistance is dopant segregation from the grain boundary into the polysilicon grains at high temperature [5]. The resistance reduction phenomenon is repeatable and has been proposed as a polysilicon resistor trimming procedure [11].

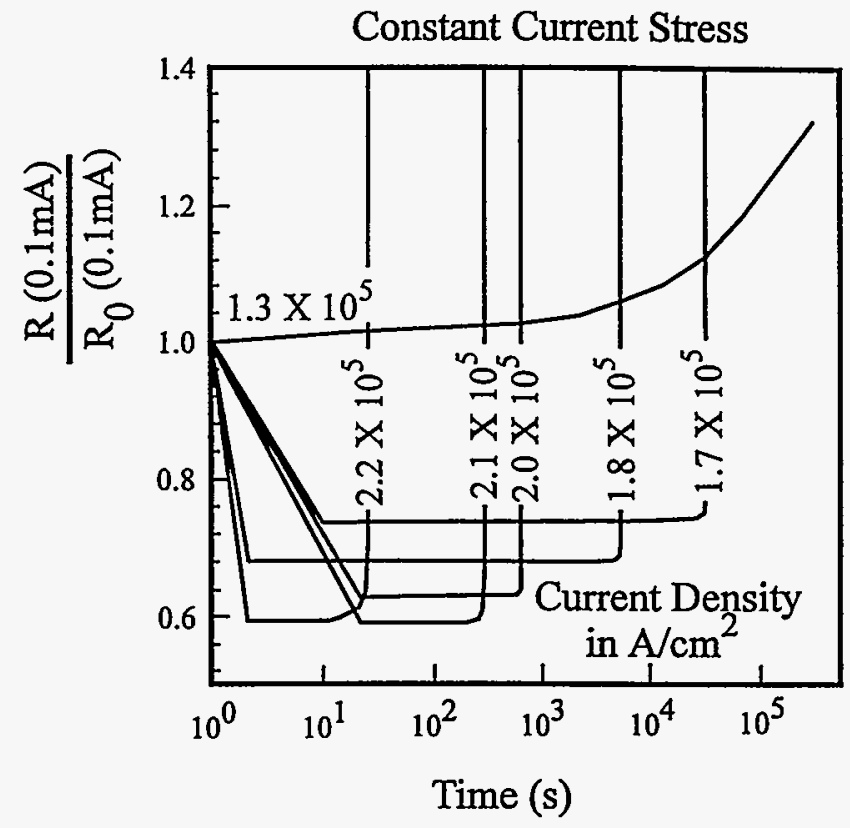

Fig. 3. Variation in relative resistance resulting from dc stressing.

At current densities above $2.0 \times 10^{5} \mathrm{~A} \mathrm{~cm}^{2}$, the resistors are visibly incandescent, making thermal gradients easily observable. Fig. 4 is an incandescent image using a $2.1 \mathrm{x}$ $10^{5} \mathrm{~A} \mathrm{~cm}^{2}$ current density. As the shape of the incandescence in Fig. 4 illustrates, the four field oxide "arms" supporting the resistor structure (Fig. 2) provide some "heat sinking" at the corners of the resistor.

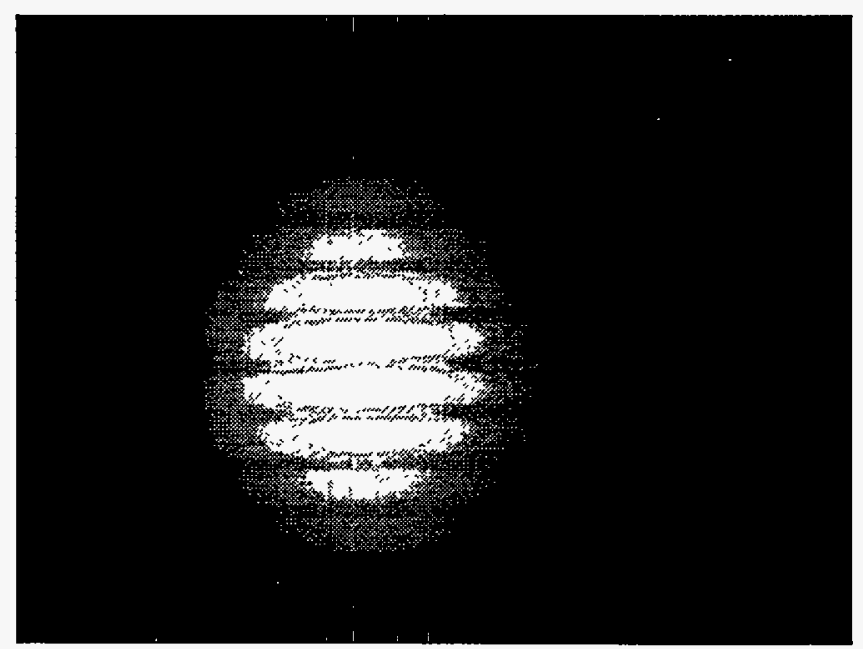

Fig. 4. Incandescent image of a polysilicon heater dc stressed at a $2.1 \times 10^{5} \mathrm{~A} / \mathrm{cm}^{2}$ current density. The field of view is similar to Fig. 2.

From power calculations, the temperature of the resistor was $\sim 1100^{\circ} \mathrm{C}$. The temperature of the polysilicon resistor during current stress is estimated by experimentally obtaining the temperature coefficient of resistance (TCR) of the polysilicon. This is performed by heating a resistor on a wafer hot-chuck and measuring the resistance versus

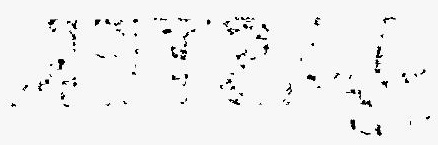




\section{DISCLAIMER}

Portions of this document may be illegible in electronic image products. Images are produced from the best available original document. 
temperature in the range between $27^{\circ} \mathrm{C}$ and $400^{\circ} \mathrm{C}$. It was noted that the resistance versus temperature characteristic is not entirely linear, especially, at temperatures exceeding $250{ }^{\circ} \mathrm{C}$. A quadratic expression is used to obtain the best possible fit to the characteristic and the coefficients were used to extrapolate to higher temperatures. The resistors are too small to use pyrometry.

The resistance versus temperature characteristic is observed to shift after stress. This shift is a function of stress current and stabilizes after the first several seconds of stress. Such a shift is probably due to stress-induced changes in the polysilicon and is analogous to changes in the TCR of metals due to impurities and microstructural scattering centers [12].

Once the resistance versus temperature characteristic was determined for a particular stress condition, the temperature was calculated by measuring the resistance increases due to the Joule heating of the film.

The second feature seen in Fig. 3 is the change in resistance with time. There is an abrupt increase in resistance with continued stressing at $1.7 \times 10^{5} \mathrm{~A} / \mathrm{cm}^{2}$ $\left(\sim 750^{\circ} \mathrm{C}\right)$ and above and a gradual increase with a $1.3 \mathrm{x}$ $10^{5} \mathrm{~A} / \mathrm{cm}^{2}\left(\sim 45^{\circ} \mathrm{C}\right)$ stress. The abrupt resistance increase occurs sooner for higher stress current densities. The abrupt increase represents catastrophic failure. The gradual increase is believed to be due to thermomigration [2] and electromigration [3] of dopants. Thermomigration of the dopants occurs when there are thermal gradients along the polysilicon resistor. The dopants diffuse from the hotter regions and cluster in the cooler areas [2]. The incandescent image shown in Fig. 4 illustrates areas of varying photon emission intensity and therefore, varying temperature (brightest $=$ hottest). The image was acquired using a standard video camera on an optical microscope. Thermomigration will tend to drive the dopant to the periphery of the resistor, away from hotter central region. Dopant electromigration in polysilicon, as in metals, is manifested by movement of dopant atoms via momentum transfer from the "electron wind" of current stressing [3]. In either the thermomigration or electromigration case, a small change in dopant distribution can greatly affect the overall resistance of a polysilicon resistor [11]. Although too small to be seen in Fig. 3, there is a gradual resistance increase under stress conditions $\geq 1.7 \times 10^{5} \mathrm{~A} / \mathrm{cm}^{2}$ after the initial resistance drop. This slight increase may be the result of electromigration with continued stress.

\section{AC Stressing}

Fig. 5 displays the resistance variation resulting from constant current density, bipolar ac stressing. Three features are evident in Fig. 5. First, as with the dc stressing shown in Fig. 3, there is an initial drop in the polysilicon resistance.

The second feature seen in Fig. 5 is an abrupt increase in resistance with continued ac stressing (following a period of slowly increasing resistance). The time to the abrupt change increases with increasing frequency. The increased time to failure with ac stressing demonstrates an electromigration effect similar to that seen in metals [13]. These results contradict earlier work that identified no electromigration effects [1]. Under ac bipolar current stress, the "electron wind" produces dopant oscillation, but will not yield net movement unless the dopant is trapped at a defect. The higher the stressing frequency, the shorter the distance a dopant travels before the electric field and the dopant direction reverse. The shorter travel distance decreases the probability that a dopant will encounter an anomaly (trap or getter site) in the polysilicon and increases the probability of observing the "time reversal" aspect of ac stressing. Fig. 6 displays the relationship between the time-to-failure and ac stressing frequency. The sample size for the $95 \%$ confidence-interval bars is between 4 and 7 , indicating a very tight distribution. The confidence-interval bars at $1 \mathrm{kHz}$ and $2 \mathrm{kHz}$ are smaller than the plotting symbol and are not visible. Above 100 $\mathrm{Hz}$ there is a monotonic increase in the time-to-failure with stress frequency. Unfortunately the test setup could not exceed a $2 \mathrm{kHz}$ frequency. Below $100 \mathrm{~Hz}$ there is a slight increase in lifetime compared to the dc stressing condition, but the lifetime is fairly "flat" over the 0.1 to $100 \mathrm{~Hz}$ range. The "flat" region probably results from stressing half periods (dopant travel distances) where the probability of interacting with an anomaly is constant.

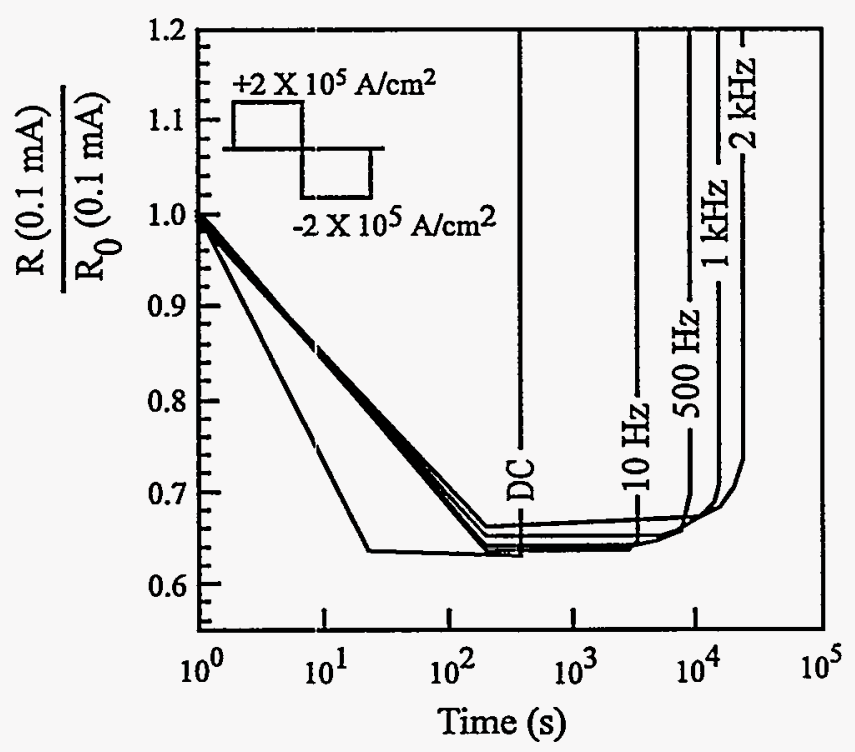

Fig. 5. Relative resistance variation resulting from ac stressing.

The third feature seen in Fig. 5 is a slight decrease in the initial resistance drop with increasing frequency. The decrease has been observed before, but was considered insignificant and possibly due to intrinsic differences in the pre-stress resistance and subsequent self-heating [1]. The frequency dependence of the initial resistance drop seen in Fig. 5 suggests a more fundamental mechanism than sample variation, possibly related to the effects of momentum transfer or a change in the resistor's Joule heating response for ac versus dc stress. The temperatures we report in this paper are based on the assumption that ac 
and dc stress of the same amplitude will produce the same temperature increase. Qualitatively, the ac and $\mathrm{dc}$ incandescent images appear identical for the same stress amplitude.

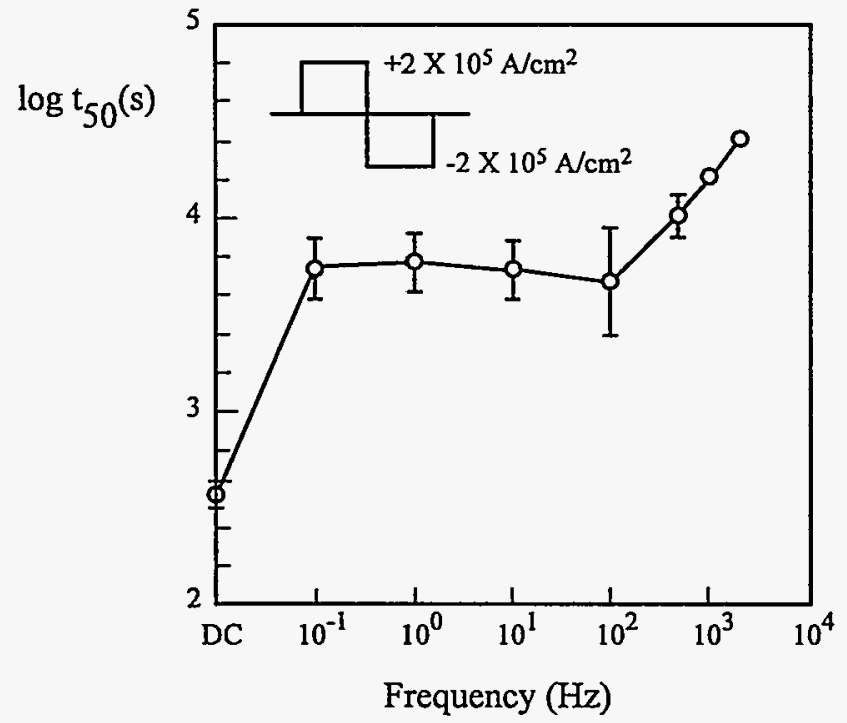

Fig. 6. Relationship between the time-to-failure and bipolar, ac stressing frequency with $a \pm 2.0 \times 10^{5}$ $\mathrm{A} / \mathrm{cm}^{2}$ applied current density.

\section{OBIC ANALYSIS}

OBIC images are obtained from polysilicon resistors to examine the changes in internal structure of the resistors with applied stress. Stress is applied using either the HP universal source and function generator described earlier or the OBIC system with the equipment described below. OBIC images are produced by monitoring the current flow through the resistors as a focused laser beam is scanned across the sample.

\section{OBIC System and Signal Generation}

A block diagram of the OBIC system is shown in Fig. 7. The scanning optical microscope (SOM) is a Zeiss Laser Scan Microscope. A $5 \mathrm{~mW}, 633 \mathrm{~nm}$, HeNe laser is used. A pair of galvanometer-driven mirrors move the laser spot across the field of view in a $512 \times 512$ pixel raster. A photomultiplier detector is used to produce reflected light images.

Some electrical stimulus of the polysilicon resistors is performed in the SOM system. This stimulus is provided by a Keithley 236 source measurement unit and an HP8111A function generator. Electrical connections to the packaged resistors are selected using a simple switch box.

The OBIC technique uses the polysilicon resistor as the detector. OBIC signals are obtained by measuring the current from an unbiased polysilicon resistor as the SOM laser beam was scanned across the structure. A GW Electronics Type 31 current amplifier is used in our experiments. The laser injects no charge, but does produce electron-hole pairs in the polysilicon. Random recombination of the electron-hole pairs produces no OBIC signal, but non-random recombination, which occurs in the presence of a Fermi level gradient (e.g. local electric field), generates an OBIC signal across the resistor terminals. No bias is applied to the resistor during OBIC imaging, so all current flow is generated by Fermi level gradients. The OBIC currents $(\sim 10 \mathrm{pA})$ are about 5 orders of magnitude smaller than those normally used in OBIC imaging and require a correspondingly higher amplifier gain. Slow scanning and frame averaging are performed to compensate for the increased noise associated with higher amplifier gain. A 10 second per frame scan rate and averaging of 8 to 10 frames are used to obtain the OBIC images.

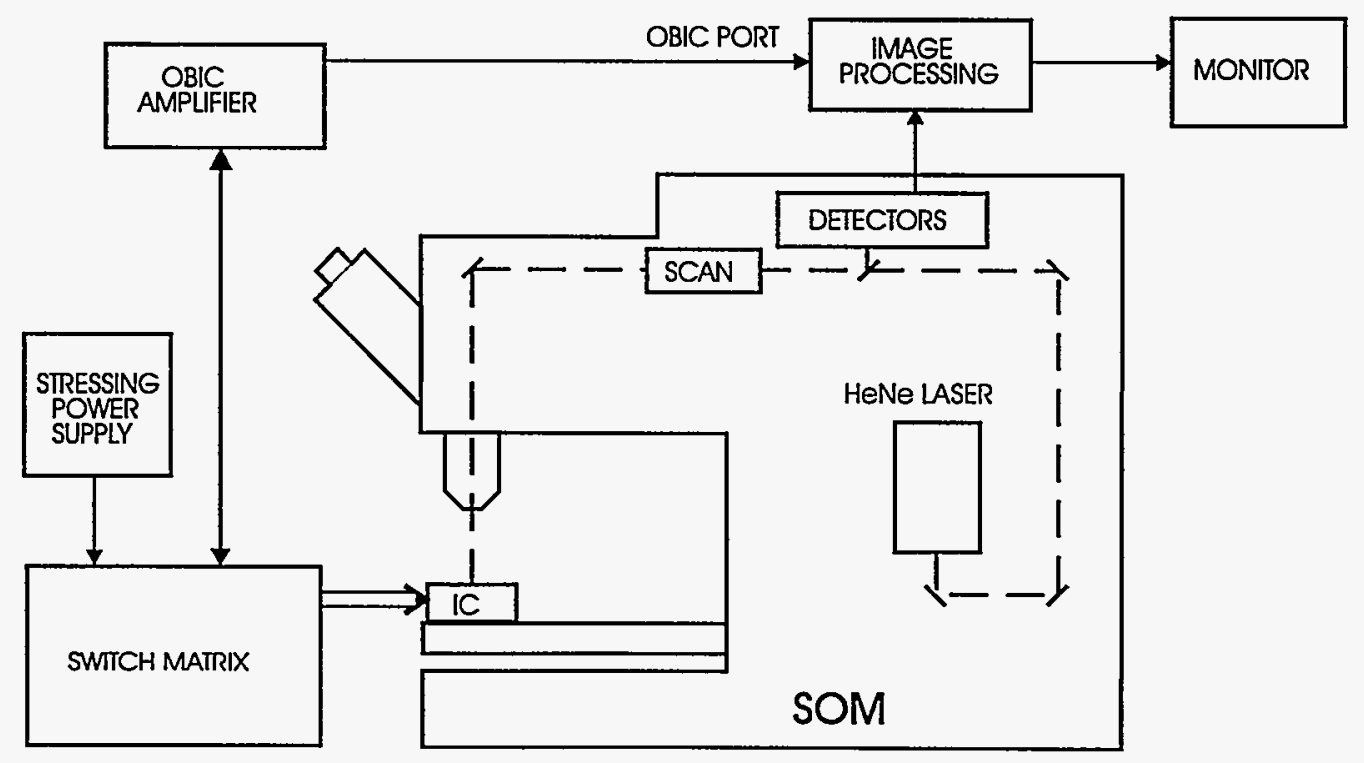

Fig. 7. Block diagram of the OBIC system. 


\section{OBIC Results on Stressed Polysilicon Resistors}

The OBIC imaging system described above is used to examine polysilicon resistors after stress with dc, bipolar ac, and mixed ac/dc currents. The stress applied to structures for OBIC analysis is current ramped from zero $\mathrm{mA}$ to the target stress current over $\sim 20$ seconds. The ramped current reduces the thermal shock to the polysilicon resistor under high stress conditions. If ramping is not used or too fast, melting of the encapsulating oxide and the mismatch in thermal expansion between the polysilicon and oxide [14] produces non-uniform oxide reflow on the surface of the polysilicon resistor (Fig. 8). The reflow obscures the OBIC image. Current ramping over 20 seconds is used for all resistor stressing except the dc results presented in Fig. 3, where 2 second ramps are used (using no ramping occasionally produced immediate catastrophic failure). No OBIC imaging is performed on the samples for Fig. 3, so the oxide reflow is not a concern. As a check. the resistance variation from dc stressing with 20 second current ramping was examined. The results are identical to those shown in Fig. 3.

DC Stressed Polysilicon Resistors: An example of OBIC analysis applied to a dc stressed, thermally-isolated polysilicon resistor is shown in Fig. 9. The resistor shown had been dc stressed at $2.1 \times 10^{5} \mathrm{~A} / \mathrm{cm}^{2}$ for 100 seconds after the current ramp. From power calculations, the temperature of the resistor is $\sim 1100^{\circ} \mathrm{C}$ under these stress conditions. The resistance value before stress is $2.2 \mathrm{k} \Omega$. After 100 seconds of stress the resistance value is $1.4 \mathrm{k} \Omega$. It is noted that the resistance drop actually occurs in less than 10 seconds. The resistance increases slowly during the subsequent stressing prior to failure. There is an overall bright to dark shading from top to bottom in the OBIC image of the resistor in Fig. 9. The shading is produced by the difference in resistance path from current generating areas on the resistor to the OBIC amplifier.

A schematic of OBIC signal generation is shown in Fig. 10. The top of the resistor is connected to the input of the OBIC amplifier. Currents produced in this area have a lower ohmic path to the amplifier and produce a brighter signal. The bottom of the resistor is connected to ground. Currents produced towards the bottom of the resistor have a lower ohmic path to ground and appear dark. At several locations along the length of the polysilicon resistor there are abrupt bright to dark contrast changes. The signals in these locations result from local gradients in the Fermi level.

An incandescent image of the polysilicon resistor in Fig. 9 is shown in Fig. 4. Note that the transition regions of bright to dark in the incandescent image align well with the OBIC contrast variations. This is consistent with the dopant thermomigration described above. The dopants are believed to diffuse from warmer areas, abruptly slowing their motion when coming in contact with a cooler region. Electromigration would also follow the thermal profile, with faster dopant motion in the hotter regions of the resistor. The diffusion (depletion) and subsequent clustering (accumulation) of dopant yield a varying Fermi level along the polysilicon resistor and therefore give rise to OBIC image contrast.

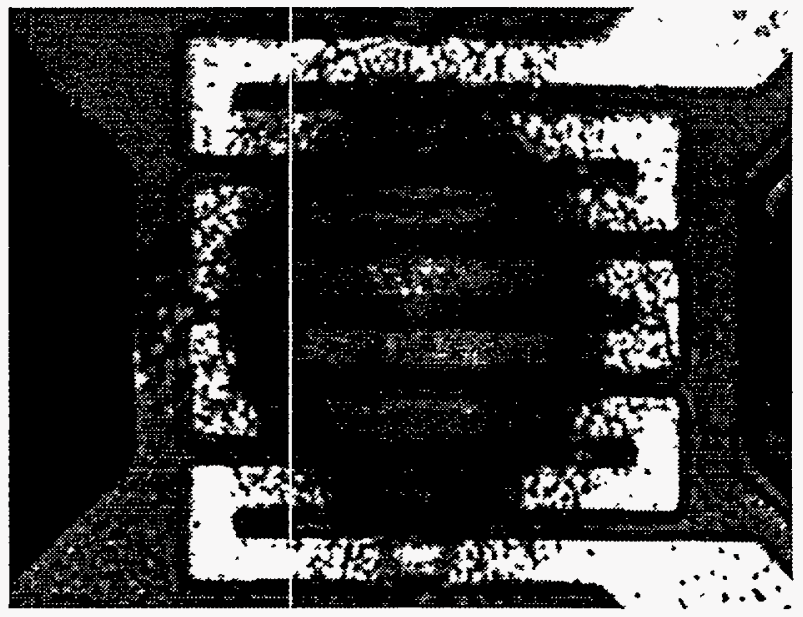

Fig. 8. Non-uniform oxide reflow produced by thermal shock. Note the similarity in shape to Fig. 4 .

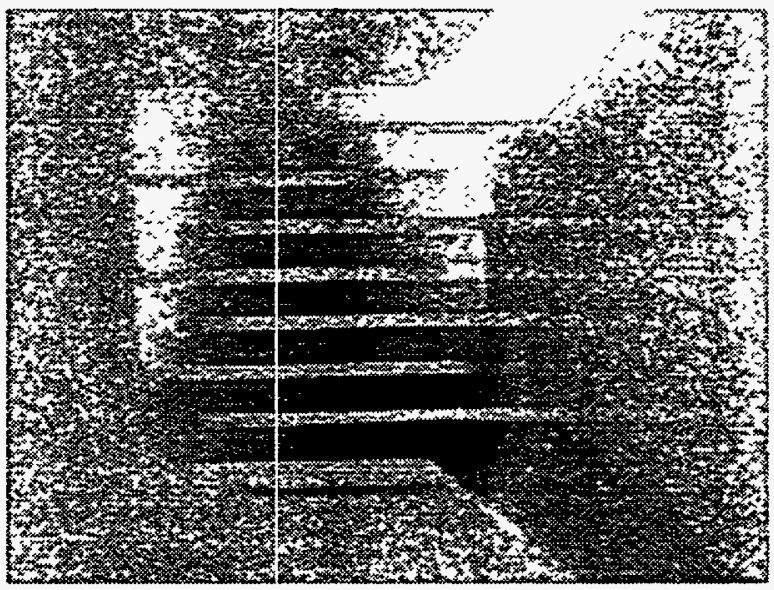

Fig. 9. OBIC image of a polysilicon resistor dc stressed at high temperature $\left(-1100^{\circ} \mathrm{C}\right)$ for 100 seconds.

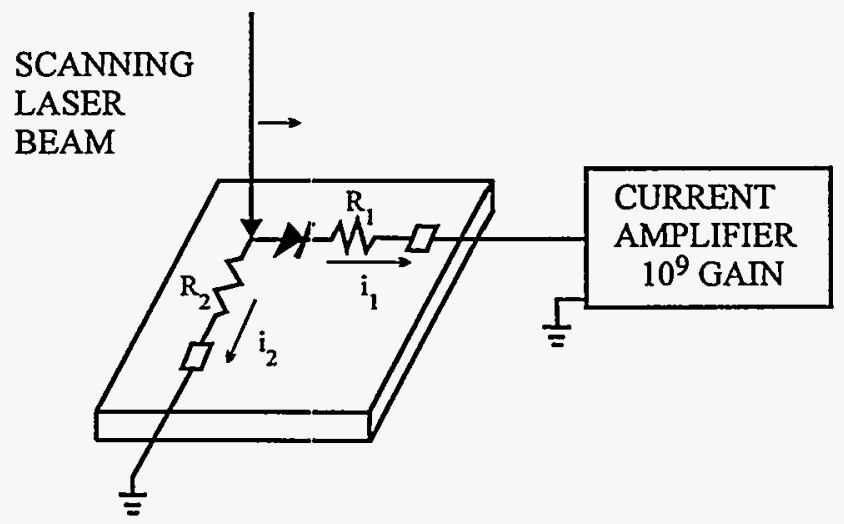

Fig. 10. Schematic of OBIC signal generation from a stressed polysilicon resistor. The diode represents a local Fermi level gradient. 
DC stress at lower current densities did not produce the initial drop in resistance, but did yield increased resistance for longer stress times. Fig. 11 is an OBIC image of a resistor $\mathrm{dc}$ stressed at $1.1 \times 10^{5} \mathrm{~A} / \mathrm{cm}^{2}$ for 48 hours. The resistance increases by $6 \%$ over this period $(2.08 \mathrm{k} \Omega$ to 2.20 $\mathrm{k} \Omega$ ). The calculated temperature of the resistor is $-170^{\circ} \mathrm{C}$ under these conditions. The OBIC contrast in Fig. 11 is reduced and is distributed differently as compared to Fig. 9. The areas of contrast change (accumulation-depletion regions) occur along the left-to-right lengths of the polysilicon resistors, similar to electromigration effects observed at $180^{\circ}$ turns in metal test structures.

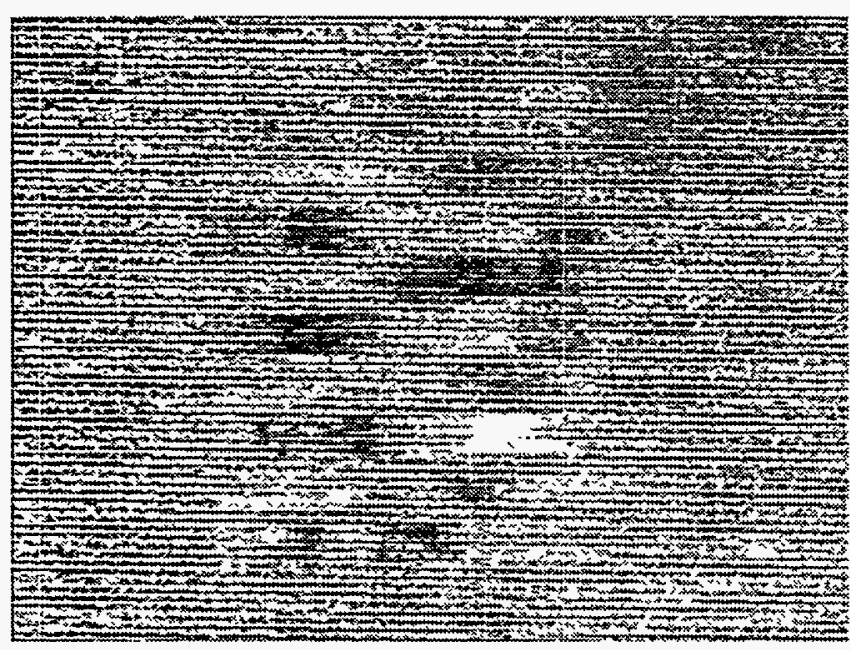

Fig. 11. OBIC image of a polysilicon resistor dc stressed at low temperature $\left(-170^{\circ} \mathrm{C}\right)$ for 48 hours.

Fig. 12 is an OBIC image of the resistor in Fig. 11 before stressing. There are some subtle contrast variations along the unstressed resistor, indicating the presence of Fermi level gradients, but the OBIC signals are weaker (and therefore a noisier image is produced) than those observed for stressed resistors.

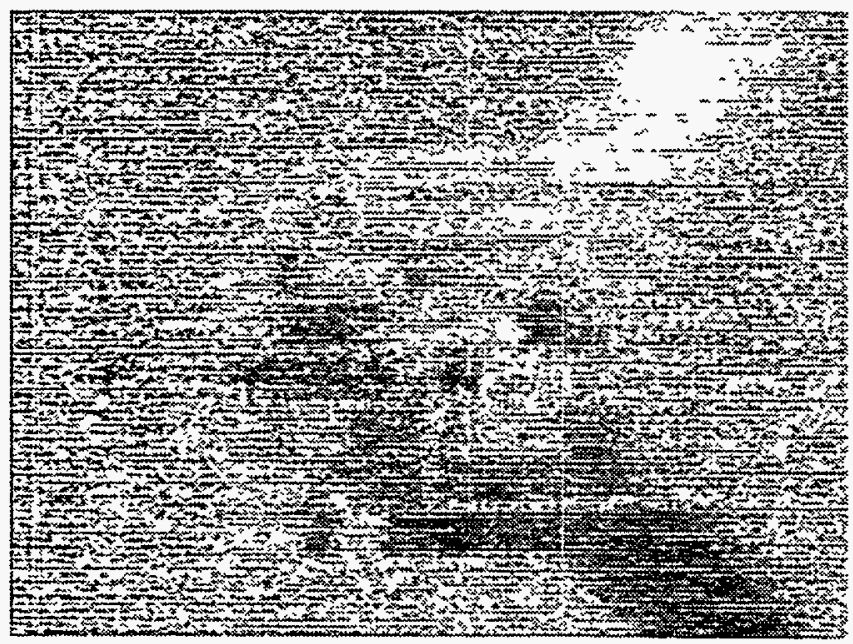

Fig. 12. An OBIC image of the resistor shown in Fig. 11 before any stressing.
Fig. 13 shows an OBIC image of a resistor dc stressed similarly to the resistor in Fig. 11 , but the current direction is reversed. Note that the bright to dark contrast variations occur in the same locations as in Fig. 11 and are not reversed as might be expected. The implications of this are addressed in the Discussion.

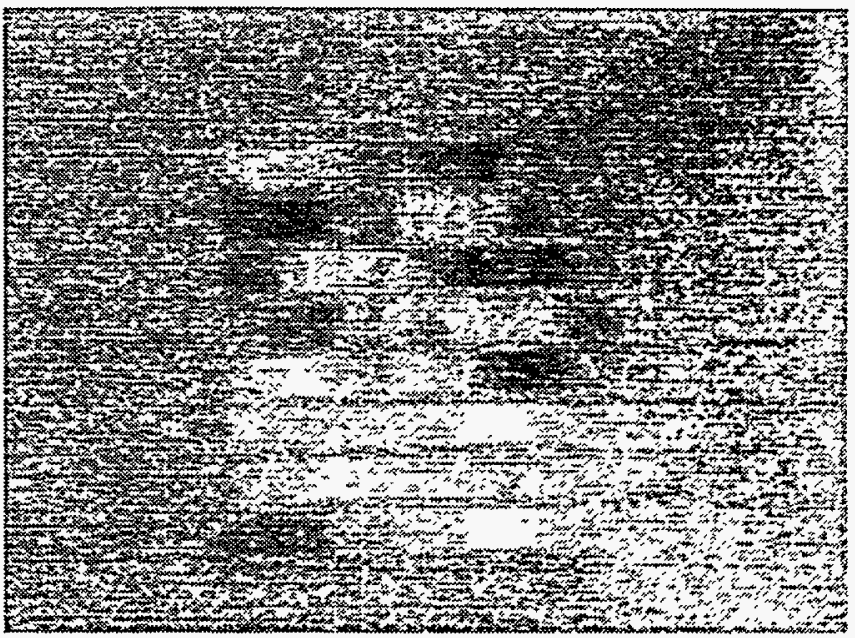

Fig. 13. OBIC image of a polysilicon resistor stressed similarly to the resistor shown in Fig. 11, but with the current direction reversed.

Fig. 14 is an OBIC image of a dc stressed resistor (2.1 $\mathrm{x}$ $10^{5} \mathrm{~A} / \mathrm{cm}^{2}, \sim 1100^{\circ} \mathrm{C}$ ) immediately before open circuit failure. The onset of failure is marked by a period of random (in time), incremental increases $(-50 \Omega)$ in resistance that persist for approximately 30 seconds before failure. A relatively strong OBIC signal is indicated by an arrow in Fig. 14. This is the site of resistor failure after subsequent stressing. Optical inspection of this site showed no anomalies immediately before failure. The strong OBIC signal probably results from variations in dopant around a developing void. Thermal gradients produced by the scanning laser at a void may also be contributing to the enhanced signal [15].

The overall OBIC signals in Fig 14. are stronger than those observed in the other resistors shown, resulting in less image noise. The relative increase in overall OBIC signal is consistent with the greater stress "damage" (greater dopant clustering) of this "about-to-fail" resistor.

AC Stressed Polysilicon Resistors: An OBIC image of an ac stressed resistor is shown in Fig. 15. The stress conditions are similar to those of the resistor in Fig. $9(2.1 \mathrm{x}$ $10^{5} \mathrm{~A} / \mathrm{cm}^{2}$ ), but the stress is applied as a $1 \mathrm{kHz}, \pm 2.1 \times 10^{5}$ $\mathrm{A} / \mathrm{cm}^{2}$, bipolar square wave for 100 seconds. $\mathrm{AC}$ incandescent images show the same heating effects as seen with dc images at $2.1 \times 10^{5} \mathrm{~A} / \mathrm{cm}^{2}$. As before, the temperature under these stress conditions is $\sim 1100^{\circ} \mathrm{C}$. As with dc stressing, the initial resistance is $2.2 \mathrm{k} \Omega$, which decreases to $1.3 \mathrm{k} \Omega$ after 100 seconds of stress. The OBIC image in Fig. 15 shows the overall resistance signal (shading) seen earlier, but none of the clustering of Fermı level gradients. The fact that an OBIC signal is generated 


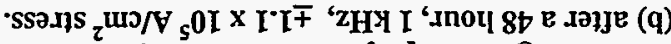

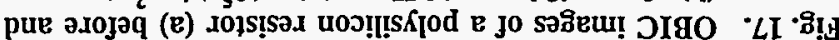
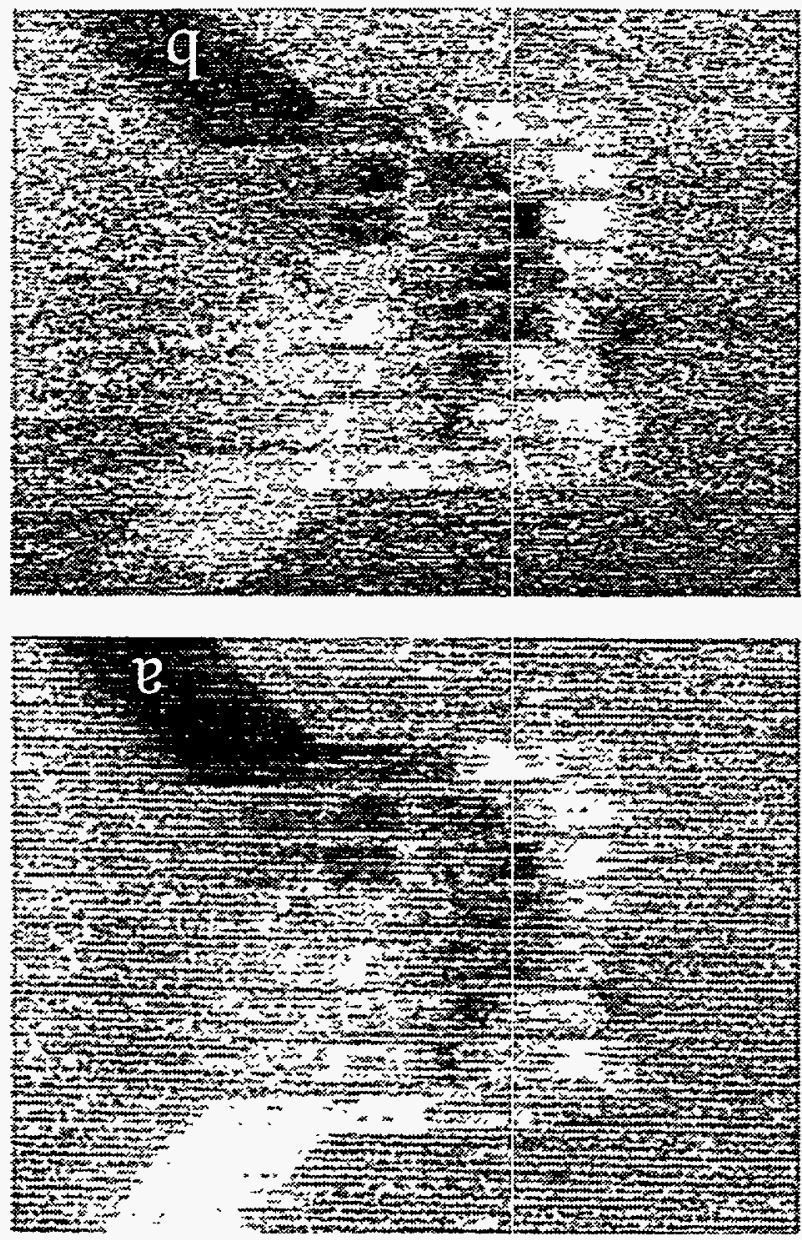

$D_{0} 0 \varepsilon L \sim$ je ssadps

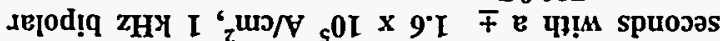

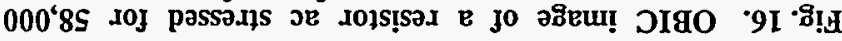

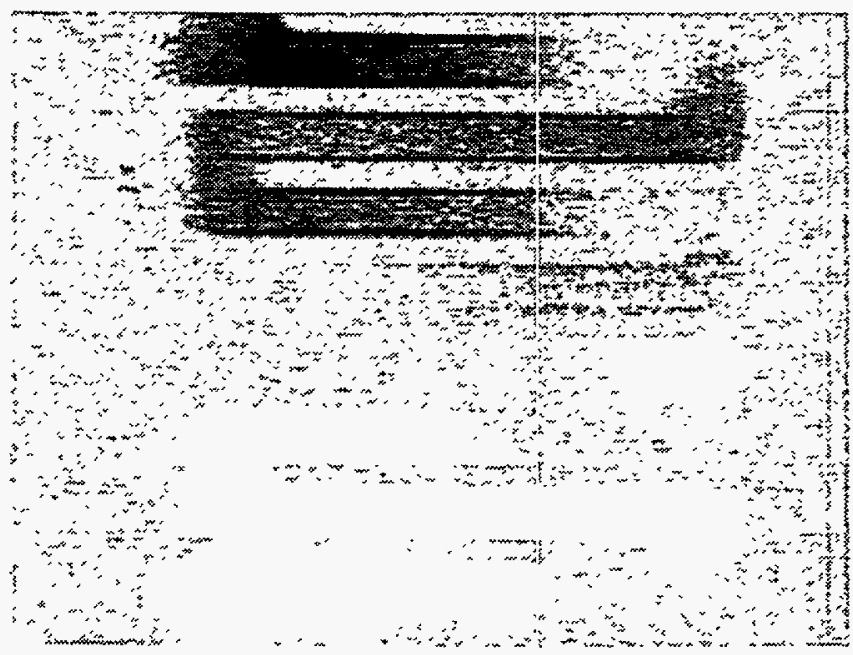

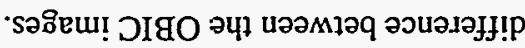

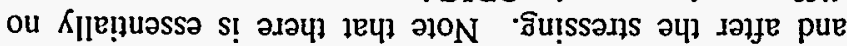

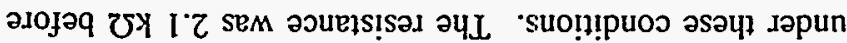

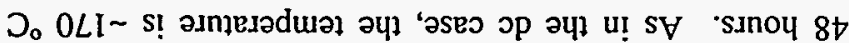

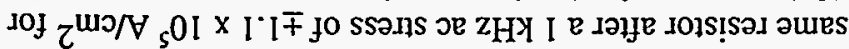

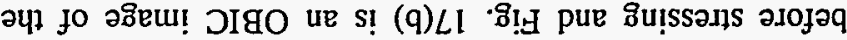

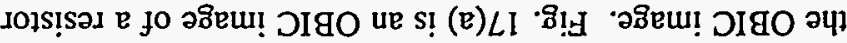

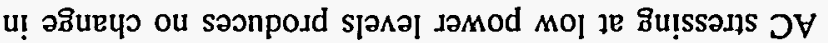

- uวəs วq ueว

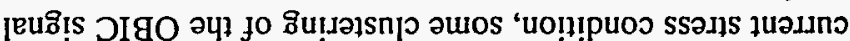

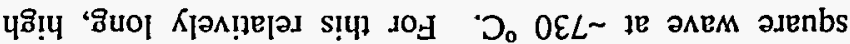

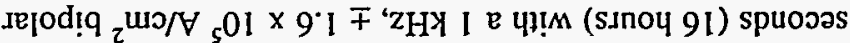

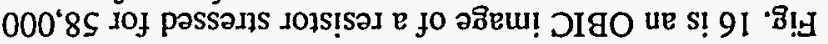

'Spuojos 00I

IOJ (2

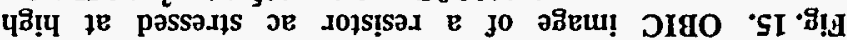

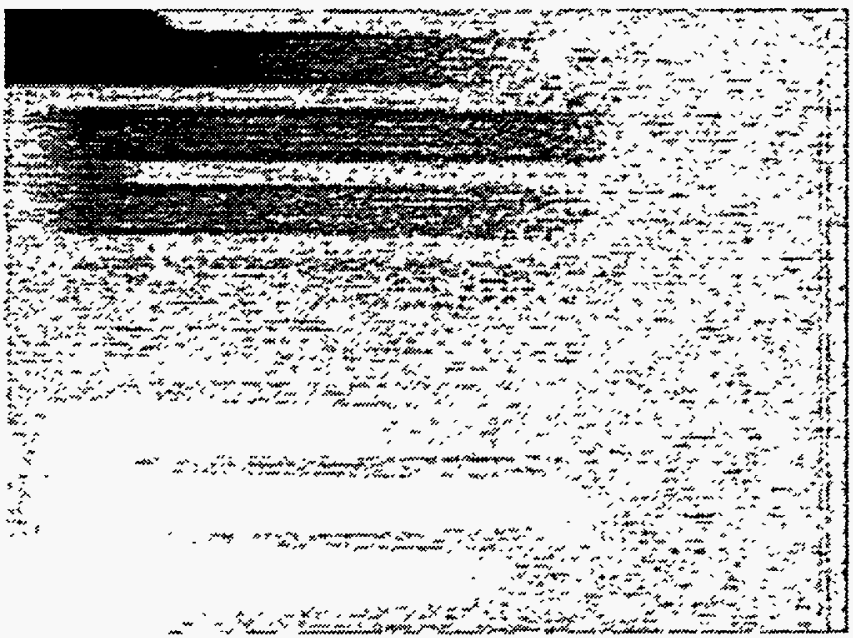

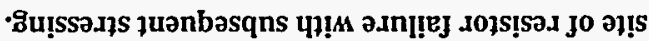

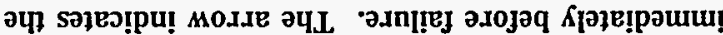

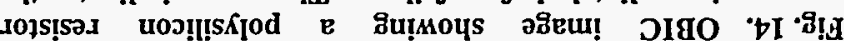

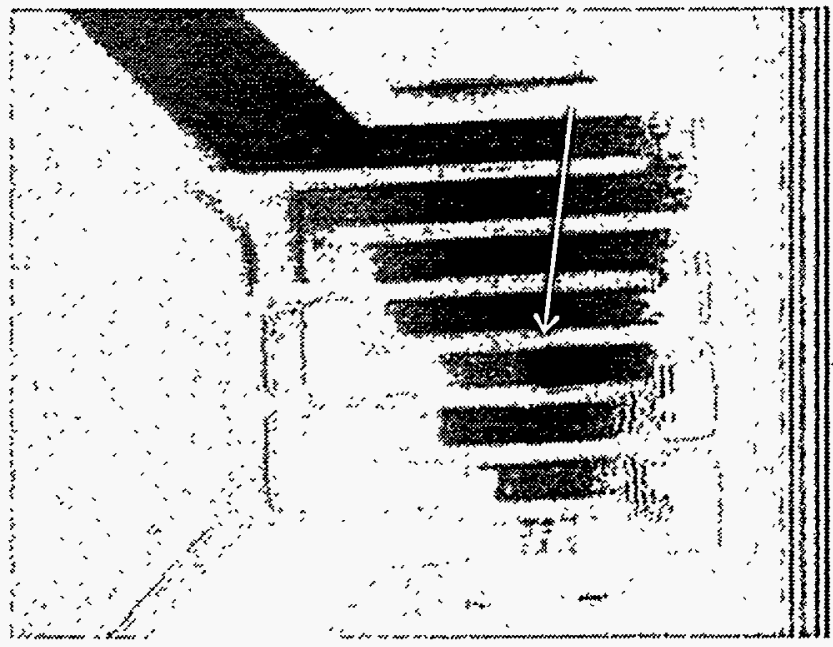

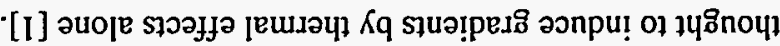

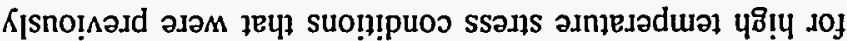

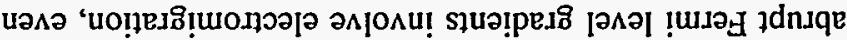
әч1 8u!

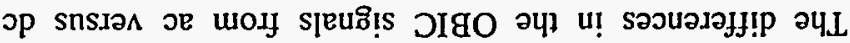

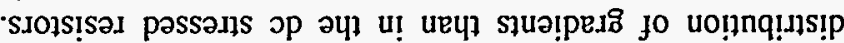

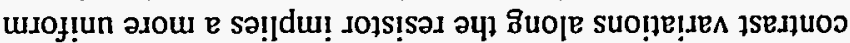

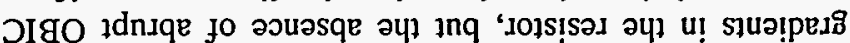

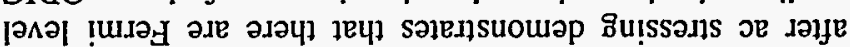


Mixed Stressing of Polysilicon Resistors: AC stressing of a polysilicon resistor after dc stressing of the same magnitude and time produced no additional clustering in the OBIC images. However, dc stressing after ac stressing produced OBIC images resembling the dc stressed example. In both cases, mixing of ac and dc stressing produced net $\mathrm{dc}$ results, as would be expected for electromigration phenomena.

\section{TEM ANALYSIS}

The microstructure of the polysilicon resistors was investigated with transmission electron microscopy (TEM). Cross-sectional samples were prepared of both a stressed $\left(2.0 \times 10^{5} \mathrm{~A} / \mathrm{cm}^{2} \mathrm{dc}\right.$ current for approximately 100 seconds $)$ and an unstressed resistor. The sections of the two samples were oriented to slice through all the fingers of each resistor. Fig. 18(a) is representative of the microstructure of the unstressed sample, and Fig. 18(b) shows the microstructure of one of the center fingers (which experienced the greatest amount of heating) of the stressed resistor. Both the stressed and unstressed polysilicon exhibit columnar grains with the grain size on the order of the layer thickness, $0.4 \mu \mathrm{m}$. Comparison of the TEM images from all the fingers of the two resistors shows no appreciable difference in microstructure between the unstressed polysilicon resistor and a resistor stressed at 2.0 $\times 10^{5} \mathrm{~A} / \mathrm{cm}^{2}\left(\sim 1100^{\circ} \mathrm{C}\right)$.

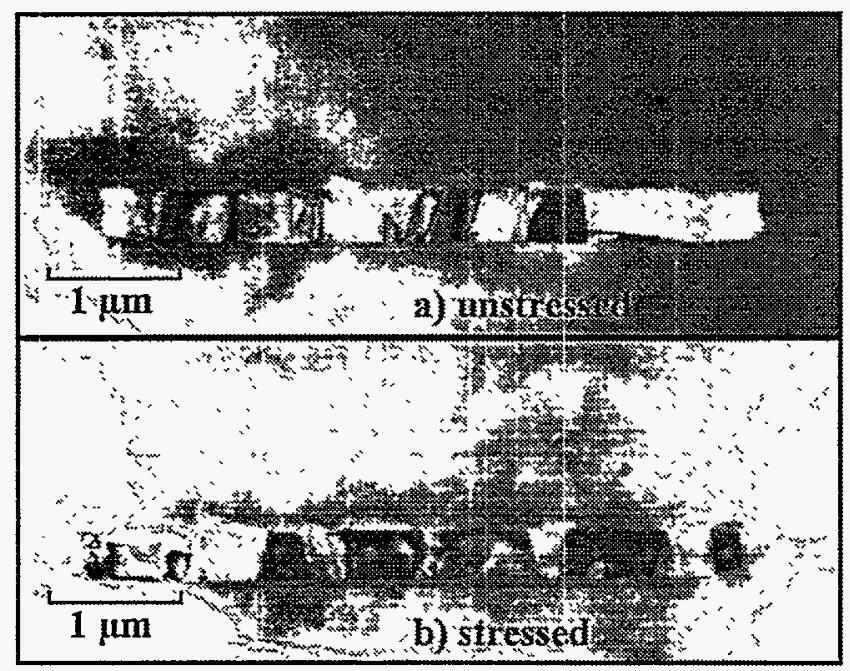

Fig. 18. TEM cross sections of an (a) unstressed and (b) stressed polysilicon resistor. The stress condition was $2.0 \times 10^{5} \mathrm{~A} / \mathrm{cm}^{2}\left(-1100^{\circ} \mathrm{C}\right)$ for $\sim 100$ seconds.

\section{DISCUSSION}

OBIC imaging and conventional electromigration testing present a consistent picture of thermally-isolated polysilicon degradation resulting from high temperature, elevated current stress. The effects of dopant clustering during dc stressing are observed as areas of changing OBIC contrast. The areas of OBIC contrast are consistent with regions of thermal gradients as seen in the incandescent images. Note that one would also expect electromigration effects to follow the thermal profile. That is, electromigration occurs faster in hotter regions. However, the polysilicon degradation under high stress conditions is not totally driven by thermal processes. The ac stressing produces a similar reduction in resistance, but the subsequent abrupt resistance increase (catastrophic failure) with continued stress occurs at a later time. OBIC images of ac stressed samples illustrate an increase in OBIC signal, but fewer of the abrupt contrast changes observed with dc stress. The differences in OBIC signals between dc and ac stress occur even though the thermal gradients are similar, as evidenced by the incandescent images. Clearly, an electromigration-type momentum transfer contributes to dopant motion in the polysilicon, even at very high temperatures.

The increase in time-to-failure with ac stress is also consistent with electromigration-type effects. In a defect free crystal, an ac stimulus provides a net zero momentum transfer over the period of the stressing signal. In a polycrystalline resistor, which does contain defects, the longer the period of ac stress, the greater the probability that a dopant atom will interact with an anomaly and not be able to return to its original state under electric field reversal. The "flat" region from 0.1 to $100 \mathrm{~Hz}$ in Fig. 6 probably represents stressing periods (dopant travel distances) where the probability of interacting with an anomaly is constant.

A previous study [1] attributed the abrupt drop in polysilicon resistance at high temperatures to recrystallization of the polysilicon, but did not include a microstructural examination. The TEM results obtained in this study show that appreciable grain growth does not occur during stressing, and thus another mechanism is responsible for the drop in resistance. The roles of both carrier trapping and dopant trapping at the grain boundaries have been examined for electrical conduction in polysilicon [5]. Both phosphorous and boron segregate to polysilicon grain boundaries at relatively lower temperatures. The reduction in carrier concentration (and hence increased resistivity) at lower temperatures is due to this segregation effect. When the dopant segregates to the grain boundary it becomes electrically inactive and the resistivity increases. Conversely, at higher temperatures the segregation is less and the carrier concentration rises. This result is consistent with our finding that the polysilicon resistance drops upon heating above $-800{ }^{\circ} \mathrm{C}$ and that the resistance drop is greater the higher the stress temperature. The saturation observed above $2.1 \times 10^{5} \mathrm{~A} / \mathrm{cm}^{2}$ in Fig. 3 is also consistent with reference [5], which shows that the increase in carrier concentration as a function of temperature saturates around $1000{ }^{\circ} \mathrm{C}$.

OBIC imaging and electrical testing at reduced temperature and current presents a consistent trend as well. At low temperatures (current densities) there is little or no dopant segregation. This is displayed by the absence of resistance decrease during electrical testing. There is also no strong OBIC contrast such as that seen with high temperature stressing. Low temperature dc stressing does produce an increase in resistance over an extended time. 
The OBIC images of low temperature, dc stressed resistors show areas of accumulation and depletion along the length of the resistor. Low temperature ac stressing produces no increase in resistance and no changes in OBIC contrast.

The reliability implications of these effects are straightforward. Lifetime is improved by operating polysilicon resistors under ac bias and increased frequency (up to $2 \mathrm{kHz}$ examined). Even at very high temperatures $\left(\sim 1100{ }^{\circ} \mathrm{C}\right)$ momentum transfer mechanisms are not overwhelmed by thermomigration. AC operation retards the development of Fermi level gradients and the accompanying increases in resistance which lead to failure. Similar heating can be achieved with both ac and dc operation, so no heater performance loss should be realized. Broad band infrared emission may have detectable variations in intensity at the ac driving frequency. These at-frequency variations should be researched, but even if they exist, acquiring data at a frequency different than the driving frequency or filtering data at the driving frequency compensates for any effects.

In addition to ac biasing, a gradual increase (over several seconds) in temperature should be used for high temperature operation. The ramp up avoids the thermal and mechanical stressing that damages the passivation layer.

The following unexplained experimental results define two areas for future work. First, there is the slight decrease in resistance drop with increasing frequency after initial ac stressing. The same stressing currents should produce similar thermal profiles and therefore the same amount of dopant segregation and resistance decrease. The fact that the resistance does not have as great a decrease at higher frequencies indicates a reduced amount of dopant segregation. The segregation may be affected by thermal and mechanical relaxation times, net momentum transfer, and other mechanisms not considered here.

Second, the fact that the accumulation and depletion of dopant is independent of current direction during low temperature, dc stressing has not been explained. This may be similar to electromigration, in that a void or hillock nucleation site will produce a void or hillock when stressed, independent of the "electron wind" direction. Similarly, areas of the polysilicon resistor may act as getters for dopant atoms under low temperature stressing, producing accumulation and depletion regions independent of the net momentum direction. The differences between the dc and ac stressing results indicate that the dopant atoms must have a net non-zero momentum transfer to produce OBIC contrast (accumulate in the getter regions), but the thermal, mechanical and material properties that produce the gettering regions have not been identified.

\section{CONCLUSIONS}

The application of small signal OBIC imaging to the examination of stressed polysilicon resistors demonstrates, for the first time, that the expected changes in Fermi level with stress can be directly observed. The OBIC technique permits nondestructive localization of internal polysilicon material variations that have no observable morphologic signatures.
Electromigration effects have been observed in OBIC images and in time-to-failure measurements for high temperature conditions. Polysilicon resistor reliability can be increased by ac operation under moderate frequency conditions. This will not impact polysilicon heater performance but may produce an ac intensity transient in spectroscopy applications.

\section{ACKNOWLEDGMENTS \& DISCLAIMER}

The authors thank Dan Barton for his assistance in sample preparation and Tom Headly, Chuck Hills, and Tom Chavez for TEM analysis. The authors also thank Richard Anderson and Jerry Soden for their careful review of the manuscript. This work was supported in part by the National Semiconductor Metrology Program at the National Institute of Standards and Technology; not subject to copyright. This work was also supported by the U.S. Department of Energy under contract number DE-AC04-94AL85000.

Commercial equipment, instruments, and computer programs may be identified to specify the procedure adequately. This does not imply recommendation or endorsement by NIST or Sandia, nor does it imply that the equipment or program is the best available for the purpose.

\section{REFERENCES}

[1] N.R. Swart and A. Nathan, "Reliability study of polysilicon for microhotplates," in Proc. IEEE Solid State Sensor and Actuator Workshop, June 13-16, 1994, pp. 119-122.

[2] C.H. Mastrangelo, J.H. Yeh, and R.S. Muller, "Electrical and optical characteristics of vacuum-sealed polysilicon microlamps," IEEE Trans. Elect. Devices, Vol. 39, No. 6, June 1992, pp. 1363-1375.

[3] J.R. Lloyd, M.R. Polcari, and G.A. MacKenzie, "Observation of electromigration in heavily doped polycrystalline silicon thin films," Appl. Phys. Lett., Vol. 36, March 1980, pp. 428-430.

[4] M. Parameswaran, A.M. Robinson, D.L. Blackburn, M. Gaitan, and J. Geist, "Micromachined thermal radiation emitter from a commercial CMOS process," IEEE Electron Device Letters, Vol. 12, No. 2., Feb 1991, pp. 57-59.

[5] M.M. Mandurah, K.C. Saraswat, and C.R. Helms, "Dopant segregation in polycrystalline silicon," J. Appl. Phys., Vol. 51, No. 11, Nov. 1980, pp. 5755-5763.

[6] K.S. Wills, T. Lewis, G. Billus, and H. Hoang, "Optical beam induced current applications for failure analysis of VLSI devices," in Proc. Int. Symp. Testing and Failure Anal. (ISTFA), 1990, pp. 21-26.

[7] E. Zaroni, G. Spiazzi, G.D. Libera, B. Bonati, M. Muschitello, and C. Canali, " Detection and Localization of gate oxide shorts in MOS transistors by optical-beaminduced current," IEEE Trans. Elect. Devices, Vol. 38, No. 2, Feb. 1991, pp. 417-419.

[8] C. Tomovich, "MOSIS - A gateway to silicon", IEEE Circuits and Devices, Vol. 4, No. 2, 1988, pp. 22-23. 
[9] C. D. Ouwens and H. Heijligers, "Recrystallization processes in polycrystalline silicon," Appl. Phys. Lett., Vol. 26, No. 10, 1975, pp. 569-571.

[10] J.Y.W. Seto, "The electrical properties of polycrystalline silicon films," Journal of Appl. Phys., Vol. 46, No. 12, 1975, pp. 5247-5254.

[11] S. Das and S.K. Lahiri, "Electrical trimming of ionbeam-sputtered polysilicon resistors by high current pulses," IEEE Trans. Elect. Devices, Vol. 41, No. 8, August 1994, pp. 1429-1434.

[12] Mott, N. F., and Jones, H., The Theory of the Properties of Metals and Alloys, Dover Publications, Inc. New York, 1958.

[13] D.K. Liew, N. W. Cheung, and C. Hu, "Projecting interconnect electromigration lifetime for arbitrary current waveforms," IEEE Trans. Elect. Devices, Vol. 37, 1990, pp. 1343-1351.

[14] J.J. Sniegowski, H. Guckel, and T.R. Christenson, "Performance characteristics of second generation polysilicon resonating beam force transducers," in Proc. IEEE Solid-State Sensor and Actuator Workshop, June 1990, pp. 9-12.

[15] T. Koyama, Y. Mashiko, M. Sekine, and H. Koyama, "New non-bias optical beam induced current (NB-OBIC) technique for evaluation of $\mathrm{Al}$ interconnects," in Proc. Int. Reliability Phys. Symp. (IRPS), 1995.

\section{DISCLAIMER}

This report was prepared as an account of work sponsored by an agency of the United States Government. Neither the United States Government nor any agency thereof, nor any of their employees, makes any warranty, express or implied, or assumes any legal liability or responsibility for the accuracy, completeness, or usefulness of any information, apparatus, product, or process disclosed, or represents that its use would not infringe privately owned rights. Reference herein to any specific commercial product, process, or service by trade name, trademark, manufacturer, or otherwise does not necessarily constitute or imply its endorsement, recommendation, or favoring by the United States Government or any agency thereof. The views and opinions of authors expressed herein do not necessarily state or reflect those of the United States Government or any agency thereof. 